\title{
THE DIMENSION OF INVERSE LIMIT AND $N$-COMPACT SPACES
}

\author{
M. G. CHARALAMBOUS
}

\begin{abstract}
For each $k=1,2, \ldots, \infty$, we construct a normal $N$-compact space $X$ with $\operatorname{dim} X=k$, where $\operatorname{dim}$ denotes covering dimension, which is the limit space of a sequence of zero-dimensional Lindelöf spaces.
\end{abstract}

Let $X$ be the limit space of an inverse sequence $\left(X_{n}, f_{n m}\right)$. In [1], we showed that $\operatorname{dim} X$ can be positive even if $X$ is normal and $X_{n}$ is Lindelöf and zero dimensional for each $n$ in $N$, the set of natural numbers. In this paper we continue investigating the behaviour of covering dimension under inverse limits. We generalise the construction in [1] to obtain, for each $k=1,2, \ldots, \infty$, an inverse sequence $\left(X_{n}, f_{n m}\right)$ of zero-dimensional Lindelöf spaces with limit space $X$ such that $X$ is normal and $\operatorname{dim} X=\operatorname{Ind} X=k$, where Ind denotes large inductive dimension. The space $X$ is, in addition, first countable, locally compact, countably paracompact and collectionwise normal. Recall that a space is called $N$-compact if it is the inverse limit of countable discrete spaces. Every zero-dimensional Lindelöf space is $N$-compact, and 80 is the inverse limit of $N$-compact spaces. It follows that $X$ is $N$-compact. $N$-compact spaces of positive covering dimension have previously been constructed in $[5,6]$ and also $[7,8]$, but our space $X$ seems to be the first example showing that $N$-compact spaces can have infinite dimension. ${ }^{1}$

In this paper, all spaces are Tychonoff. The usual metric on the Cantor set $C$ is denoted by $d$. I denotes the unit interval, $\omega(c)$ the first ordinal of cardinality $c$, the cardinality of the continuum, and $|X|$ the cardinality of a set $X$.

For standard results in Dimension Theory we refer to [4].

1. Preliminaries. The construction of the spaces $X$ and $X_{n}$ in this paper is only slightly more complicated than that of the corresponding spaces in [1]. In both papers, we draw from the techniques employed in $[2,7,8]$. The constructions in [1] made use of Wage's complete separable metric $\rho$ on $C$ which has the following properties: (a) the $\rho$-topology is finer than the usual topology on $C,(\mathrm{~b})$ every $\rho$-Borel set of $C$ is $d$-Borel, and (c) every $\rho$-open set disjoint from a certain fixed $\rho$-closed set $E$ has boundary of cardinality $c$. The constructions in the present paper are based on the existence of a separable metric $e$ on $C$ with the properties enumerated in the following result.

Proposition 1. For each $k=1,2, \ldots, \infty$, there exists a separable metric $e$ on $C$ with $d \leq e$ and $k$ pairs of disjoint $e$-closed sets $E_{i}, F_{i}, i=1,2, \ldots, k$, such that

Received by the editors September 21, 1981.

1980 Mathematics Subject Classification. Primary 54F45, 54G20.

Key words and phrases. Normal, Lindelöf, paracompact, $N$-compact space, covering and inductive dimension.

${ }^{1}$ The referee has informed the author that $R$. Engelking and E. Pol have recently constructed, for each $k \in\{1,2, \ldots, \infty\}$ a Lindelöf, zero-dimensional space $X=X(k)$ such that $\operatorname{dim} X^{2}=k$. 
(1) every uncountable e-closed and every nonempty e-open subset of $C$ has cardinality $c$.

(2) Whenever $L_{i}, i=1, \ldots, k$, is an e-partition between $E_{i}$ and $F_{i}$, then

$$
\left|\bigcap_{i=1}^{k} L_{i}\right|=c .
$$

(3) $\operatorname{dim}(C, e)=k$.

$N . B$. The condition $d \leq e$ implies that the $e$-topology is finer than the usual topology on $C$.

ProOF. Let $Y=C \times I^{k}$ and $\pi=Y \rightarrow C$ the canonical projection. Let $\mathcal{G}$ be the collection of all subsets $G$ of $Y$ such that $|\pi(G)|=c$ and $G$ is either open or closed. Note that $|\mathcal{G}|=c$ and we may choose an enumeration $\left\{G_{\alpha}: \alpha<\omega(c)\right\}$ of $\mathcal{G}$ such that for each $G$ in $\mathcal{G}, G=G_{\alpha}$ for $c$ ordinals $\alpha<\omega(c)$. For each $\alpha<\omega(c)$, since $\left|\pi\left(G_{\alpha}\right)\right|=c$, we can choose by transfinite induction, a point $x_{\alpha}$ in $\pi\left(G_{\alpha}\right)$ so that $x_{\alpha} \neq x_{\beta}$ for $\alpha \neq \beta$. Next, we define a function $f: C \rightarrow I^{k}$ as follows. If $x=x_{\alpha}$ for some $\alpha<\omega(c)$, we choose $f(x)$ so that $(x, f(x))$ is in $G_{\alpha}$. If not, we set $f(x)=0$.

Let $X$ be the subspace $\{(x, f(x)): x \in C\}$ of $Y$. Then $\pi: X \rightarrow C$ is bijective and continuous. Consider an uncountable closed set $E$ of $X$. Then $E=F \cap X$ for some closed subset $F$ of $Y$. Now the closed set $\pi(F)$ of $C$ is uncountable and hence has cardinality $c$, so that $F$ is in $\mathcal{G}$. If $A=\left\{\alpha<\omega(c): F=G_{\alpha}\right\}$, then $E$ contains $\left\{\left(x_{\alpha}, f\left(x_{\alpha}\right): \alpha \in A\right\}\right.$, and since $x_{\alpha} \neq x_{\beta}$ for $\alpha \neq \beta$, and $|A|=c$, we have $|E|=c$. Similarly, every nonempty open subset of $X$ has cardinality $c$. In fact, $|G \cap X|=c$ for every $G$ in 9 .

Let $A_{i}, B_{i}, i=1, \ldots, k$, be the pairs of opposite faces of $I^{k}$. Let $U_{i}, V_{i}$ be open sets of $Y$ such that $C \times A_{i} \subset U_{i}, C \times B_{i} \subset V_{i}$ and $\bar{U}_{i} \cap \bar{V}_{i}=\emptyset$. Set $E_{i} \doteq X \cap \bar{U}_{i}$, $F_{i}=X \cap \bar{V}_{i}, i=1, \ldots, k$, and suppose $L_{i}$ is a partition in $X$ between $E_{i}$ and $F_{i}$. Then there exist disjoint open sets $G_{i}, H_{i}$ of $Y$ with $E_{i} \subset G_{i}, F_{i} \subset H_{i}$ and $X-\left(G_{i} \cup H_{i}\right) \subset L_{i}$. Let $P_{i}=U_{i} \cup\left(G_{i}-\bar{V}_{i}\right), Q_{i}=V_{i} \cup\left(H_{i}-\bar{U}_{i}\right)$ and $M_{i}=$ $Y-\left(P_{i} \cup Q_{i}\right)$. Then $M_{i}$ is a partition in $Y$ between $C \times A_{i}$ and $C \times B_{i}$ with $M_{i} \cap X \subset L_{i}$. It follows that $\bigcap_{i=1}^{k} M_{i}$ contains at least one point from $\{x\} \times I^{k}$ for each $x$ in $C$. Thus $\left|\pi\left(\bigcap_{i=1}^{k} M_{i}\right)\right|=c$, which implies that

$$
\left|\bigcap_{i=1}^{k} M_{i} \cap X\right|=\left|\bigcap_{i=1}^{k} L_{i}\right|=c .
$$

Now, by the Otto-Eilenberg characterisation of covering dimension [4, Theorem 1.7.9], $\operatorname{dim} X \geq k$ and, since $X$ is a subspace of the $k$-dimensional space $Y$, we have $\operatorname{dim} X=k$.

Finally, to complete the proof of Proposition 1, it suffices to let, for $x, y$ in $C$, $e(x, y)$ denote the usual Euclidean distance between the points $(x, f(x))$ and $(y, f(y))$.

We shall need the following result, which is the analogue for the metric $e$ on $C$ of the well-known theorem on the existence of Bernstein sets in a complete separable metric space.

Proposition 2. There is a partition $A_{1}, A_{2}, A_{3}, \ldots$ of $C$ such that $\left|A_{i} \cap F\right|=c$ for each $i$ in $N$ and each uncountable e-closed set $F$ of $C$. 


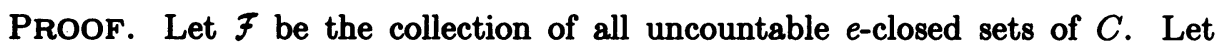
$\left\{F_{\alpha}: \alpha<\omega(c)\right\}$ be an enumeration of $\mathcal{F}$ such that for each $F$ in $\mathcal{F}, F=F_{\alpha}$ for $c$ ordinals $\alpha<\omega(c)$. For each $\alpha$, by Proposition $1,\left|F_{\alpha}\right|=c$ and thus we can choose by transfinite induction points $x_{\alpha 1}, x_{\alpha 2}, \ldots$ in $F_{\alpha}$ so that $x_{\alpha n} \neq x_{\beta m}$ if $(\alpha, n) \neq(\beta, m)$. It now suffices to let $A_{i}=\left\{x_{\alpha i}: \alpha<\omega(c)\right\}$ for $i=2,3, \ldots$, and $A_{1}=C-\bigcup_{i=2}^{\infty} A_{i}$.

N.B. It follows from Proposition 1(1), that each $A_{i}$ is $e$-dense in $C$.

2. The construction. In the sequel, $k$ denotes a fixed number of the set $\{1,2, \ldots, \infty\}, e$ the metric on $C$ given by Proposition 1 , and $A_{1}, A_{2}, \ldots$ the partition of $C$ provided by Proposition 2.

Let $\left\{\left(S_{\alpha 1}, S_{\alpha 2}, \ldots\right): \alpha<\omega(c)\right\}$ be the collection of all sequences of countable subsets of $A_{1}$ with $\left|\bigcap_{i=1}^{\infty} \bar{S}_{\alpha i}^{e}\right|=c$. Since

$$
\left|A_{1} \cap \bigcap_{i=1}^{\infty} \bar{S}_{\alpha i}^{e}\right|=c,
$$

for each $\alpha<\omega(c)$, we can choose $x_{\alpha}$ in $A_{1} \cap \bigcap_{i=1}^{\infty} \bar{S}_{\alpha i}^{e}$ and a sequence $\left\{x_{\alpha n}\right\}$ in $A_{1}$ so that $e\left(x_{\alpha}, x_{\alpha n}\right)<\frac{1}{n},\left\{x_{\alpha n}\right\}$ contains infinitely many points from each $S_{\alpha i}$, $x_{\alpha} \neq x_{\beta}$ for $\alpha \neq \beta$ and $x_{\alpha n} \triangleleft x_{\alpha}$, where $\triangleleft$ is some well-ordering on $C$ of the same type as $\omega(c)$. Let $A=\left\{x_{\alpha}: \alpha<\omega(c)\right\}$.

For each $x$ in $C$, we construct a decreasing sequence $\left\{B_{m}(x): m \in N\right\}$ of countable subsets of $C$ containing $x$ as follows. For $x \notin A$, we set $B_{m}(x)=\{x\}$. On $A$ we define $B_{m}$ by transfinite induction with respect to $\triangleleft$ by setting for each $\alpha<\omega(c)$

$$
B_{m}\left(x_{\alpha}\right)=\left\{x_{\alpha}\right\} \cup \bigcup\left(B_{n}\left(x_{\alpha n}\right): n \geq 2 m\right) .
$$

It follows by transfinite induction that if $y \in B_{m}(x)$, then $B_{n}(y) \subset B_{m}(x)$ for some $n$ in $N$, so that $\left\{B_{m}(x): m \in N\right\}$ constitutes a local base of open sets at $x$ with respect to some first countable, locally countable topology $\tau$ on $C$. It is readily seen that $B_{m}(x)$ is $d$-closed and has e-diameter $\leq \frac{1}{m}$. Hence $\tau$ is finer than the e-topology on $C, B_{m}(x)$ is $\tau$-clopen and ind $(C, \tau)=0$. In fact, each infinite sequence in $B_{m}(x)$ has an accumulation point in $B_{m}(x)$, so that $B_{m}(x)$ is $\tau$-compact and $(C, \tau)$ is locally compact. In the sequel, $X$ denotes the space $(C, \tau)$.

Next, for each $i$ in $N$, we define a first countable topology $\tau_{i}$ finer than the $d-$ topology on $C$ by defining at each point $x$ a local base $\left\{B_{m}^{i}(x): m \in N\right\}$ consisting of a decreasing sequence of $d$-closed sets containing $x$ as follows. If $x$ is in $\bigcup_{j=i+1}^{\infty} A_{j}$, we let $\left\{B_{m}^{i}(x): m \in N\right\}$ be a decreasing sequence of $d$-clopen sets of $C$ forming a local $d$-base at $x$. Otherwise, we let $B_{m}^{i}(x)=B_{m}(x)$. Clearly, $\operatorname{ind}\left(C, \tau_{i}\right)=0$. In the sequel, $X_{i}$ stands for the space $\left(C, \tau_{i}\right)$. It is readily verified that $\left(X_{i}, f_{i j}\right)$, where $f_{i j}: X_{j} \rightarrow X_{i}$ is the identity mapping, $i<j$, constitutes an inverse limit sequence with limit space $X$.

The short proof of the following result is almost identical with the proof of Claim 1 of [1]. We give it here for completeness.

Claim 1. For each $i$ in $N, X_{i}$ is a Lindelöf space with $\operatorname{dim} X_{i}=0$.

ProOF. Since every open set of $X_{i}$ containing a point of $A_{i+1}$ is a $d$-open neighbourhood of that point, for any open cover $\mathcal{U}$ of $X_{i}$, we can choose $d$-open sets $G_{n}, n$ in $N$, such that each $G_{n}$ is contained in some member of $U$ and $A_{i+1} \subset$ $G=\bigcup_{n=1}^{\infty} G_{n}$. Since $X_{i}-G$ is an e-closed set of $C$ which does not intersect $A_{i+1}$, 
it follows from Proposition 2 that $X_{i}-G$ is countable. This clearly implies that $X_{i}$ is Lindelöf and hence, since ind $X_{i}=0, \operatorname{dim} X_{i}=0$.

Claim 2. $X$ is normal, countably paracompact and collectionwise normal.

ProOF. Since $X-A_{1}$ is a clopen discrete subspace of $X$, it suffices to show that $A_{1}$ is normal, countably paracompact and collectionwise normal. Note that if $B_{1}, B_{2}, \ldots$ are closed subsets of $A_{1}$ with $\bigcap_{i=1}^{\infty} B_{i}=\emptyset$, then $\bigcap_{i=1}^{\infty} \bar{B}_{i}^{e}$ is countable. For suppose that $\bigcap_{i=1}^{\infty} \bar{B}_{i}^{e}$ is uncountable and hence has cardinality $c$. For each $i$ in $N$, let $S_{i}$ be a countable $e$-dense subset of $B_{i}$. Then for some $\alpha<\omega(c)$, $\left(S_{1}, S_{2}, \ldots\right)=\left(S_{\alpha 1}, S_{\alpha 2}, \ldots\right)$ so that, by the definition of the topology $\tau, x_{\alpha}$ is an accumulation point of each $S_{i}$. Hence $x_{\alpha} \in \bigcap_{i=1}^{\infty} B_{i}$, a contradiction.

Let $E, F$ be disjoint closed sets of $A_{1}$. Then $A_{1} \cap \bar{E}^{e} \cap \bar{F}^{e}$ is a countable zero set of $A_{1}$ and, using the fact that $A_{1}$ is locally countable, we can construct a countable cozero set $Z$ of $A_{1}$ containing it. Let $P$ be a cozero and $Q$ a zero set of $A_{1}$ with $A_{1} \cap \bar{E}^{e} \cap \bar{F}^{e} \subset P \subset Q \subset Z$. Since ind $X=0$, then ind $Z \leq 0$ and, since $Z$ is countable and therefore Lindelöf, we have $\operatorname{dim} Z \leq 0$. Hence there exists a clopen set $Y$ of $Z$ such that $A_{1} \cap \bar{E}^{e} \cap \bar{F}^{e} \subset Y \subset P$. Clearly, $Y$ is a closed subset of $Q$, and thus $Y$ is a countable clopen subset of $A_{1}$. Now $Y$ is Lindelöf and therefore normal, and hence there exist disjoint open subsets $G_{1}, H_{1}$ of $Y$ with $E \cap Y \subset G_{1}$ and $F \cap Y \subset H_{1}$. Also, there exist disjoint e-open subsets $G_{2}, H_{2}$ of $A_{1}-Y$ with $A_{1} \cap \bar{E}^{e}-Y \subset G_{2}$ and $A_{1} \cap \bar{F}^{e}-Y \subset H_{2}$. Finally, $G=G_{1} \cup G_{2}$ and $H=H_{1} \cup H_{2}$ are disjoint open subsets of $A_{1}$ with $E \subset G$ and $F \subset H$. Thus $A_{1}$ is normal.

Let $\left\{B_{i}: i \in N\right\}$ be a decreasing sequence of closed sets of $A_{1}$ with $\bigcap_{i=1}^{\infty} B_{i}=0$. $A_{1}$ is countably paracompact if there exists a decreasing sequence $\left\{W_{i}: i \in N\right\}$ of open sets of $A_{1}$ with $B_{i} \subset W_{i}$ and $\bigcap_{i=1}^{\infty} W_{i}=0[3$, Corollary 5.2.2]. Now $B=$ $A_{1} \cap \bigcap_{i=1}^{\infty} \bar{B}_{i}^{e}$ is a countable subset of the locally countable space $A_{1}$ and hence it is contained in a countable open set $Y=\left\{y_{1}, y_{2}, \ldots\right\}$ of $A_{1}$, where $y_{i} \neq y_{j}$ for $i \neq j$. We may assume $B_{i} \cap B \subset\left\{y_{i}, y_{i+1}, \ldots\right\}$. Let $\left\{G_{i}: i \in N\right\}$ be a decreasing sequence of e-open sets of $A_{1}-B$ such that $A_{1} \cap \bar{B}_{i}^{e}-B \subset G_{i}$ and $\bigcap_{i=1}^{\infty} G_{i}=\emptyset$, and put $W_{i}=G_{i} \cup\left\{y_{i}, y_{i+1}, \ldots\right\}$. Then $\left\{W_{i}: i \in N\right\}$ is a decreasing sequence of open sets of $A_{1}$ with $B_{i} \subset W_{i}$ and $\bigcap_{i=1}^{\infty} W_{i}=\emptyset$. Hence $A_{1}$ is countably paracompact.

To prove that the normal space $A_{1}$ is collectionwise normal, it suffices to show that every discrete closed subset $B$ of $A_{1}$ is countable. Suppose the contrary, and let $S$ be a countable $e$-dense subset of $B$. Then $\left|\bar{S}^{e}\right|=c$ and, for some $\alpha<\omega(c)$, $(S, S, \ldots)=\left(S_{\alpha 1}, S_{\alpha 2}, \ldots\right)$, so that, by the definition of $\tau, x_{\alpha}$ is an accumulation point of $B$, contradicting the fact that $B$ is discrete. This concludes the proof of Claim 2.

Claim 3. $\operatorname{dim} X=\operatorname{Ind} X=k$.

Proof. Let $E_{i}, F_{i}, i=1, \ldots, k$, be the e-closed sets of $X$ occurring in Proposition 1. For each $i$, let $U_{i}, V_{i}$ be e-open sets of $X$ with $E_{i} \subset U_{i}, F_{i} \subset V_{i}$ and $\bar{U}_{i}^{e} \cap \bar{V}_{i}^{e}=\emptyset$, and suppose a closed subset $L_{i}$ of $A_{1}$ is a partition between $\bar{U}_{i}^{e}$ and $\bar{V}_{i}^{e}$. Write $L_{i}=M_{i} \cap N_{i}$ where $M_{i}, N_{i}$ are closed sets of $A_{1}$ with $\bar{U}_{i}^{e} \cap N_{i}=\emptyset$, $\bar{V}_{i}^{e} \cap M_{i}=\emptyset$ and $A_{1}=M_{i} \cup N_{i}$. Then it follows from the fact that $A_{1}$ is $e$-dense in $X$ that $\bar{M}_{i}^{e} \cap \bar{N}_{i}^{e}$ is an $e$-partition between $E_{i}$ and $F_{i}$. Hence, by Proposition 1 , $\left|\bigcap_{i=1}^{k} \bar{M}_{i}^{e} \cap \bar{N}_{i}^{e}\right|=c$, which implies that $\bigcap_{i=1}^{k} M_{i} \cap N_{i}=\bigcap_{i=1}^{k} L_{i} \neq 0$. Now by the Eilenberg-Otton characterisation of covering dimension we have $\operatorname{dim} X \geq k$. 
Consider next a closed subset $A$ of $A_{1}$ with $\operatorname{dim}(A, e) \leq n$. Let $E, F$ be disjoint closed subsets of $A$. Then $B=\bar{E}^{e} \cap \bar{F}^{e} \cap A$ is countable and hence is contained in a countable open set $Y$ of $A$. Clearly, $\operatorname{dim} Y \leq 0$ and hence, since also $A$ is normal, there is a clopen set $Z$ of $A$ with $B \subset Z \subset Y$. Also, there are disjoint open sets $P, Q$ of $Z$ such that $E \cap Z \subset P, F \cap Z \subset Q$ and $Z=P \cup Q$. Now $\operatorname{Ind}(A-Z, e) \leq \operatorname{Ind}(A, e) \leq n$, and so there are disjoint $e$-open sets $U, V$ of $A-Z$ such that $A \cap \overline{\bar{E}}^{e}-Z \subset U, A \cap \bar{F}^{e}-Z \subset V$ and $\operatorname{Ind}(A-(U \cup V \cup Z), e) \leq n-1$. Then $G=U \cup P, H=V \cup Q$ are disjoint open sets of $A$ with $E \subset G, F \subset H$ and Ind $(A-(G \cup H), e) \leq n-1$. It follows by induction that Ind $A \leq n$. In particular, Ind $A_{1} \leq \operatorname{Ind}(C, e)=k$ and hence, since the discrete space $X-A_{1}$ is clopen in $X$, Ind $X \leq k$. Hence, in view of the inequality $\operatorname{dim} \leq$ Ind, which holds for all normal spaces, we have $\operatorname{dim} X=\operatorname{Ind} X=k$.

\section{REFERENCES}

1. M. G. Charalambous, An example concerning inverse limit sequences of normal spaces, Proc. Amer. Math. Soc. 78 (1980), 605-607.

2. E. van Douwen, $A$ technique for constructing honest locally compact submetrizable examples, preprint.

3. R. Engelking, General topology, PWN, Warsaw, 1977.

4. _ Dimension theory, North-Holland, Amsterdam, 1978.

5. S. Mrowka, Recent results on E-compact spaces, General Topology and its Applications (Proc. Second Pittsburgh Internat. Conf.), Lecture Notes in Math., vol. 378, Springer-Verlag, Berlin and New York, 1974, pp. 298-301.

6. E. Pol and R. Pol, A hereditarily normal strongly zero-dimensional space with a subspace of positive dimension and an $N$-compact space of positive dimension, Fund. Math. 97 (1977), 43-50.

7. T. Przymusinski, On the dimension of product spaces and an example of $M$. Wage, Proc. Amer. Math. Soc. 76 (1979), 315-321.

8. M. Wage, The dimension of product spaces, Proc. Nat. Acad. Sci. U.S.A. 75 (1978), 4671-4672.

DEPARTMENT OF MATHEMATICS, UNIVERSITY OF NAIROBI, NAIROBI, P.O. BOX 30197, 